\title{
The ideal: enemy of the useful?
}

\author{
Trevor A Sheldon, Andrew F Long, Nick Freemantle, Fujian Song
}

The paper by Torgerson et al (p 48) ${ }^{1}$ is useful in that it describes a place where we would like to be, where there is perfect information on effectiveness, relevant outcome data, and reliable data on marginal costs. It also allows us the opportunity of clarifying the role of the Effective Health Care bulletins and of discussing some of the problems we have encountered over nearly three years of reviewing the literature.

The Effective Health Care bulletins were commissioned by the Department of Health at a time when it was realised that setting up the mechanisms of the internal market would not automatically result in the purchasing and provision of health care which was evidence based. The health service (and purchasers in particular) needed research based "intelligence" to inform their work. The aim of the bulletins was, "to provide valid and systematic data to aid purchasers in purchasing and providing health care ... to help decision makers in the health service make more informed decisions using the latest available information on the effectiveness of particular health interventions." ${ }^{2}$ The bulletins are based primarily on a systematic review and synthesis of published (and peer reviewed) literature on the clinical effectiveness of healthcare interventions. This is supplemented whenever possible with evidence on cost effectiveness, together with consideration of some issues surrounding the acceptability of the interventions being examined. ${ }^{3}$ They are intended to provide credible summaries of the evidence, but not guidelines, to inform choice, but not to determine decisions, and their use will reflect NHS priorities rather than set them. In this paper we address the broad issues raised by Torgerson et al.

\section{Effectiveness}

A key dimension of the quality of care is effectiveness - whether an intervention does more good than harm. Effective Health Care bulletins, by systematically reviewing the evidence on clinical effectiveness, address this issue as first among equals. Such evidence is scattered through the literature, is of variable quality, and is often difficult to interpret. By reviewing the research literature and providing focused and accessible reviews targeted at key decision makers, there is a greater likelihood that the information used by decision makers will be reliable.

Torgerson et al are right to draw attention to the need, when evaluating health care, to consider broader measures of outcome which are important to and valued by patients. However, because most research uses only those outcomes which are easy to measure or of interest to clinicians, reviews of the literature will only rarely be able to examine these broader effects. We highlighted this problem in the bulletins several times. For example, "The management of infertility should be evaluated according to the degree to which it has been successful in reducing stress, distress, or social handicap.... This could involve, for example, measures of the extent to which treatment helps couples come to terms with their childlessness." ${ }^{4}$ However, nearly all of the evidence on the effectiveness of treatments concentrated on reproductive outcomes. In our review of the surgical treatment of glue ear we recommend that "research should use broader measures of outcome than just hearing loss" and in the bulletin on stroke services we argued for "the development and use of patient and carer centred outcomes such as measures of well being, quality of life, and satisfaction." Pointing to the lack of information on these types of outcomes for interventions is useful because it identifies uncertainties about the effectiveness of treatments that need further research. Finally, when evaluating cholesterol screening we reported the evidence to show that "mass population screening and the labelling of asymptomatic patients as high risk may result in a reduction in quality of life and the adoption of a sick role in some people."

Although healthcare evaluations should explore a range of outcomes and integrate the effectiveness, outcome, and user involvement agendas, this is a very complex task which has

\section{... healthcare evaluations should explore a range of outcomes ...}

generated much debate. ${ }^{89}$ Despite the limitations in the literature, however, the information on clinical effectiveness can be useful. It enabled us to highlight, for example, that much gynaecological surgery was relatively ineffective for subfertility due to significantly blocked fallopian tubes and so reveal that these resources were being wasted ${ }^{4}$; that screening for osteoporosis was unlikely to have much impact on the number of hip fractures in elderly women ${ }^{2}$; that a proportion of operations for glue ear might be unnecessary ${ }^{5}$; that cholesterol screening was unlikely to have much effect on coronary heart disease ${ }^{7}$; that newer antidepressants were not substantially better to justify their routine first line use ${ }^{10}$; and that brief interventions can reduce alcohol consumption. ${ }^{11}$ 


\section{Economic analysis}

The Effective Health Care bulletins concentrate primarily on clinical effectiveness rather than economics because effectiveness is a necessary condition for cost effectiveness. In some of the topics reviewed so far evidence for effectiveness was so lacking that the issues of cost effectiveness were irrelevant.

There is little information on costs in the NHS and few reliable economic analyses which we have been able to incorporate in our reviews. Where the evidence exists, the bulletins attempt to provide information on costs (sometimes in the form of a cost effectiveness analysis) so that decision makers can take into account the cost implications of various actions. For example, the bulletin on treatments for depression (one of three bulletins not mentioned by Torgerson et al) gave detailed costing. ${ }^{10}$ When reviewing bone density screening we estimated the cost of starting up a bone screening and treatment programme which could then be related to the potential benefits reported. ${ }^{2}$ The costs per cycle of each element of a subfertility service (for example, in vitro fertilisation, tubal surgery, clomiphene citrate treatment) and the corresponding increase in the pregnancy rate per cycle was estimated. ${ }^{4}$ From this a purchaser can explore the relative cost effectiveness of different options. We also included the possible extra costs due to demand for neonatal intensive care services resulting from assisted conception. The review could not, nor was intended to, advise purchasers as to the level of resources (if any) to devote to subfertility services. ${ }^{4}$

Cost-benefit analysis is widely acknowledged as the most powerful of the techniques of economic evaluation. However, its use poses several theoretical and practical problems and its application is rare in healthcare evaluation. ${ }^{12}$ Torgerson et al do not provide for those topics we covered any examples of extant economic appraisals which would give purchasers further information on the economic implications of alternatives.

\section{Acceptability}

Unfortunately, the issue of acceptability of treatments to patients or users has had limited emphasis within the series so far. For example, in reviewing population based screening for osteoporosis $^{2}$ and the management of depression in primary care ${ }^{10}$ acceptability was rather narrowly represented by uptake and compliance, respectively, which may loosely reflect some aspect of the patient's perceptions of the interventions. Health outcomes are the result of a complex interaction between individual patients and their circumstances. ${ }^{8}$

The estimated uptake rate of bone density screening is one of several variables which influence the potential impact of such a programme on the level of hip fractures and was not used as some threshold measure of acceptability of the programme. ${ }^{2}$ In the context of depression, non-compliance with the drug treatment may occur for various reasons - for example, unpleasant side effects or a perceived irrelevance of drug treatment in the context of the self perceived underlying cause of the depression, or both. In the bulletin on managing depression, of necessity, we focused exclusively on compliance. ${ }^{10}$ Indeed, this was particularly important since the new selective serotonin reuptake inhibitors (SSRIs) were being marketed largely on the basis of alleged improved acceptability to patients - which our meta-analysis did not confirm. ${ }^{13}$

Given the lack of recorded information in clinical research on acceptability of treatment to patients - even side effects are poorly recorded in published papers - limited attention in the bulletins to issues of acceptability of treatment to patients and clinicians is unsurprising. From a clinical perspective compliance and uptake are critical; from a patient or user perspective the issue is

Acceptability to patients undoubtedly has a great impact on the effectiveness of treatments ...

broader, including patients' attitudes and perceptions of the clinical condition, its prognosis, and side effects of treatment. Acceptability to patients undoubtedly has a great impact on the effectiveness of treatments but has been poorly addressed within biomedical research. ${ }^{89}$

\section{Cost of illness}

Torgerson et al maintain that the choice of topics in the bulletins is critically based on a burden of illness criterion, but this is incorrect. The choice is based on three explicit criteria: the intervention has a high resource implication, its effectiveness is uncertain or disputed, and it probably has a large impact on health gain. ${ }^{3}$ Glue ear and subfertility would not have been chosen if the criterion was burden of illness. "It is the impact of changes in decision making at the margin that is the most important consideration and not simply the total volume of activity or the prevalence of the condition." 14 It is, of course, hard to adhere closely to criteria about the benefits of an intervention relative to its costs because we do not know the effectiveness and cost of the intervention before doing the research for the bulletin.

\section{Alternative interventions}

The bulletins always consider alternative interventions but will make recommendations only when the evidence has been reviewed. For example, we reviewed a range of drug and nondrug interventions for depression ${ }^{10}$ and a large number of alternative treatments for subfertility. ${ }^{4}$ We discussed the alternative of a period of watchful waiting for children with all but the most severe glue ear. ${ }^{5}$ Further, after demonstrating the lack of evidence for the effectiveness of bone screening we suggested 
that health authorities examine the effectiveness of alternative interventions, including population measures such as exercise programmes. ${ }^{2}$ However, in this case we made clear that we had reviewed only the literature on osteoporosis screening and that health authorities would have to investigate further these alternatives.

\section{Purchasing at the margin}

Issues of purchasing at the margin are given much attention by Torgerson et al, but these were addressed in the bulletins. For example, we accepted that all districts purchase some level of subfertility services and by considering the effectiveness of treatment we explored the potential gain of shifting resources among treatments. Thus, a major summary point was that "surgery is not effective for women with severely damaged fallopian tubes. In such cases resources would be more efficiently allocated to assisted conception." 4

Giving general guidance here is difficult because it is impossible to predict all the options that purchasers may be considering. Also for several interventions we examined, such as population screening for osteoporosis, there is no evidence that any component of the intervention should be bought, never mind how much.

\section{Conclusions}

Torgerson et al made several suggestions which need to be taken into account when providing advice to commissioners. Unfortunately, because of the limitations in the published research and dearth of relevant data on costs in the NHS many of these cannot yet be incorporated in reliable health technology assessments based on reviews of the evidence. Solving these problems will require a shift in the type of research that is carried out - for example, biomedical researchers need to adopt a broader perspective, using outcome criteria which incorporate patients' or users' viewpoint.

The current focus of the bulletins is justifiable because before cost effectiveness analysis, considerations of margins, and all the other elements of economic evaluation can be sensibly brought to bear on the problem the effectiveness of treatments must be established. Economists have generally been too ready to carry out economic analysis on the basis of inadequate evidence about effectiveness. ${ }^{15}$ Our priority is to provide reliable and objective evidence to inform purchasing rather than construct more complete or elaborate analyses which combine evidence with assumptions, individual judgements, or the consensus of experts.

1 Torgerson D, Ryan M, Donaldson C. Effective Health Care bulletins: are they effective? Quality in Health Carc 1995;4:48-51.

2 Effective Health Care. Screening for osteoporosis to prezent fractures. Leeds: School of Public Health, University of Leeds; Centre for Health Economics, University of York; Research Unit, Royal College of Physicians, 1992. Research Unit,
(Bulletin No 1.)

3 Long AF, Sheldon TA. Enhanced effective and acceptable purchaser decisions: overview and methods. Quality in Health Care 1992;1:74-6.

4 Effective Health Care. The management of subfertility. Leeds: School of Public Health, University of Leeds; Centre for Health Economics, Univesity of York; Research Unit, Royal College of Physicians, 1992. (Bulletin No 3.)

5 Effective Health Care. The treatment of persistent glue ear in children. Leeds: School of Public Health, University of Leeds; Centre for Health Economics, University of York; Research Unit, Royal College of Physicians, 1992.

6 Effective Health Care. Stroke rehabilitation. Leeds: School of Public Health, University of Leeds; Centre for Health Public Health, University of Leeds; Centre for Health Economics, University of York; Research

7 Effective Health Care Cholesterol: screening and treatment. Leeds: Nuffield Institute for Health, University of Leeds; Centre for Health Economics, University of York; Research Unit, Royal College of Physicians, 1993. (Bulletin No 6.)

8 Gill TM, Feinstein AR. A critical appraisal of quality-of-life measurements. FAMA 1994;272:630 1.

9 Guyatt GH, Cook DJ. Health status, quality of life, and the individual. $7 A M A$ 1994;272:619-26.

10 Effective Health Care. The treatment of depression in primary care. Leeds: School of Public Health, University of Leeds; Centre for Health Economics, University of York; Research Unit, Royal College of Physicians, 1993. Research Unit,
(Bulletin No 5.)

11 Effective Health Care Brief interentions and alcohol usc. Leeds: Nuffield Institute for Health, University of Leeds; Centre for Health Economics, Research Unit, Roval College of Physicians, 1993. (Bulletin No 7.)

12 Robinson R. Cost-benefit analysis. BMF 1993;307:924-6.

13 Song F, Freemantle N, Sheldon TA, House A, Watson P, Long $\mathrm{A}$, et al. Selective serotonin reuptake inhibitors: efficacy, acceptability, and effectiveness. A meta-analysis. BMF 1993;306:683-7.

14 Freemantle N, Sheldon TA. Prioritising resources. BMY 1993;307:896-7.

15 Freemantle N, Maynard A. Something rotten in the state of clinical and economic evaluations? Health Economics 1994;3:63-7. 